\title{
On the physics potential of ILC and CLIC
}

\section{Aleksander Filip Żarnecki* on behalf of the ILD concept group and the CLICdp collaboration}

Faculty of Physics, University of Warsaw

E-mail: filip.zarneckilfuw.edu.pl

\begin{abstract}
The International Linear Collider (ILC) and the Compact Linear Collider (CLIC) are the two options for a future high-energy, high-luminosity linear electron-positron collider. Both are expected to be built in stages, optimised for their physics potential. The main goals are the precision measurements of Higgs-boson and top-quark properties as well as direct and indirect searches for new physics Beyond Standard Model. In my talk I will review some of the latest results from both ILC and CLIC demonstrating their physics potential, pointing to similarities and complementarity of both projects.
\end{abstract}

Corfu Summer Institute 2019 "School and Workshops on Elementary Particle Physics and Gravity" (CORFU2019)

31 August - 25 September 2019

Corfù, Greece

\footnotetext{
* Speaker.
} 


\section{Introduction}

The European Strategy for Particle Physics is expected to be updated by May 2020 and will clearly guide the development of the field in the next decade. The research program of the LHC and HL-LHC is unlikely to be able to solve all the problems of particle physics not addressed in the Standard Model. There is a strong scientific case for an electron-positron collider, complementary to the LHC, that can study the properties of the Higgs boson and other particles with unprecedented precision. In particular, the worldwide community has been engaged for many years in two linear collider projects: International Linear Collider (ILC) which is under consideration to be built in Japan and Compact Linear Collider (CLIC) at CERN. This contribution reviews the physics potential of both ILC and CLIC. After the two collider projects and the experimental environments have been described, a personal selection of the physics highlights is presented, pointing to similarities and complementarity of the projects. The three pillars of the research program for future high energy $\mathrm{e}^{+} \mathrm{e}^{-}$colliders will be discussed: Higgs boson studies, top quark physics and searches for Beyond the Standard Model (BSM) phenomena. For more information the reader is referred to inputs submitted to the European Strategy for Particle Physics Update by the ILC [1] and CLIC [2] collaborations.

\section{Colliders}

\subsection{International Linear Collider}

The International Linear Collider (ILC) project is based on the technology of superconducting accelerating cavities. In the Technical Design Report (TDR) completed in 2013 [3], construction of a machine with a centre-of-mass energy of $500 \mathrm{GeV}$ and a footprint of $31 \mathrm{~km}$ was proposed, see Fig. 1, with a possible upgrade to $1 \mathrm{TeV}$. The baseline design includes polarisation for both $e^{-}$and $e^{+}$beams, of $80 \%$ and $30 \%$, respectively. The discovery of a Higgs Boson with a mass of $125 \mathrm{GeV}$ motivated the possibility of reducing initial ILC cost by starting at a centre-of-mass energy of $250 \mathrm{GeV}$ [4] followed by a $500 \mathrm{GeV}$ stage and $1 \mathrm{TeV}$ considered as the possible future upgrade, should the physics case for such an upgrade be compelling. The baseline running scenario for the staged ILC construction is presented in Fig. 2 [5]. In the assumed 22-year running period

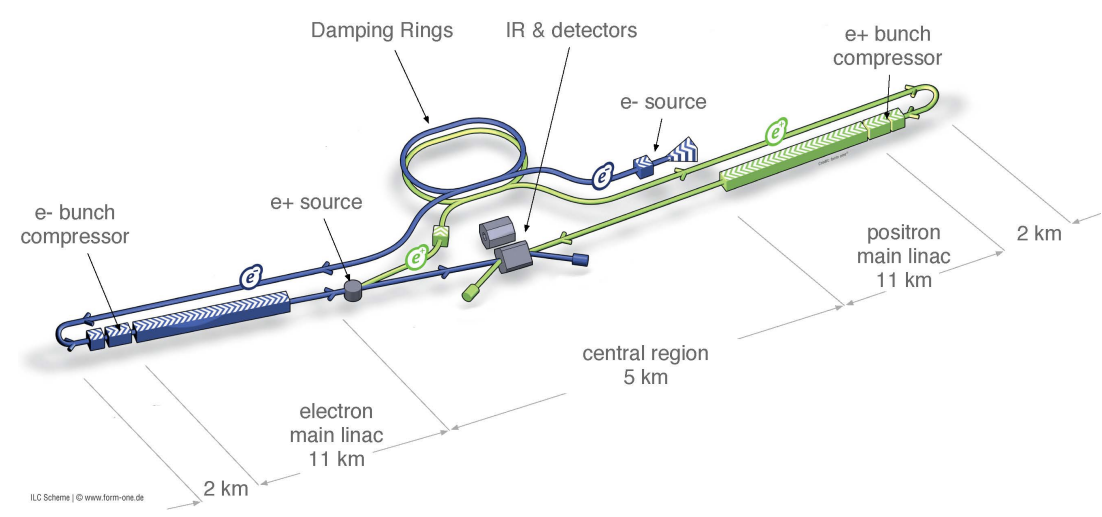

Figure 1: Schematic layout of the ILC in the $500 \mathrm{GeV}$ configuration, as described in [3]. 


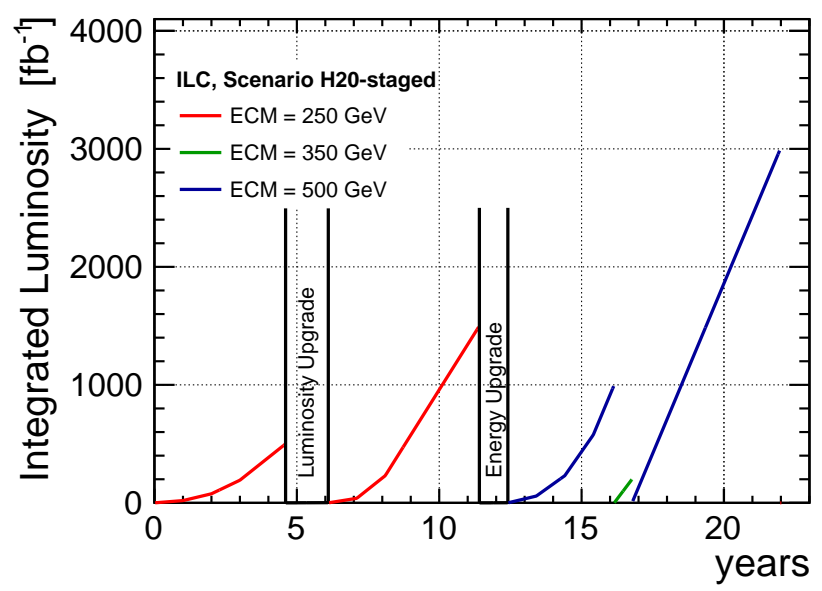

Figure 2: The nominal 22-year running program for the staged ILC, starting operation at $250 \mathrm{GeV}$ [5].

the ILC is expected to deliver the integrated luminosities of about $2 \mathrm{ab}^{-1}$ at $250 \mathrm{GeV}$ and $4 \mathrm{ab}^{-1}$ at $500 \mathrm{GeV}$, with an additional $200 \mathrm{fb}^{-1}$ collected at the top-quark pair-production threshold around $350 \mathrm{GeV}$. One has to stress that these integrated luminosities are the same as in original $\mathrm{H}-20$ proposal for ILC starting at $500 \mathrm{GeV}$ [6]. ILC is basically ready to be built with all construction issues verified. The European XFEL accelerator built with the ILC technology [3], with the nominal energy of $17.5 \mathrm{GeV}$. It started its operation in May 2017, and can be considered the largest ever accelerator prototype and resulted in full industrialisation of the cavity production. In fact, most of the European XFEL cavities already meet ILC requirements. Nevertheless, cavity design and production optimisation studies are still ongoing.

\subsection{Compact LInear Collider}

The Conceptual Design Report (CDR) for the Compact Linear Collider (CLIC) was presented in 2012 [7]. CLIC is based on the two-beam acceleration scheme, required to generate a high RF gradient of about $100 \mathrm{MV} / \mathrm{m}$, see Fig. 3. For an optimal exploitation of its physics potential the implementation plan for CLIC [8] assumes construction in three stages, with 7 to 8 years of data taking at each stage, see Fig. 4. The first stage with a footprint of $11 \mathrm{~km}$ and an energy of $380 \mathrm{GeV}$ will focus on precision Standard Model physics and is optimised for Higgs boson and top-quark measurements. The plan assumes collecting $1 \mathrm{ab}^{-1}$ at this stage, including $100 \mathrm{fb}^{-1}$ collected

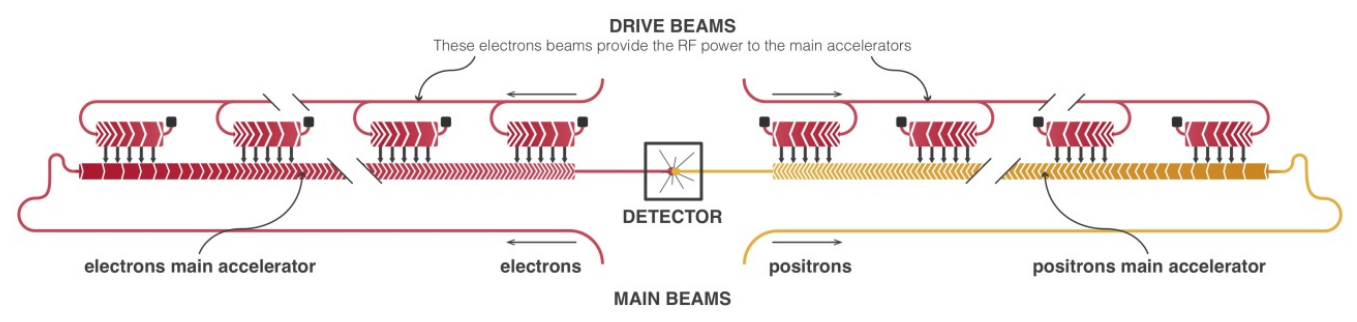

Figure 3: Schematic layout of the CLIC accelerator [9]. 


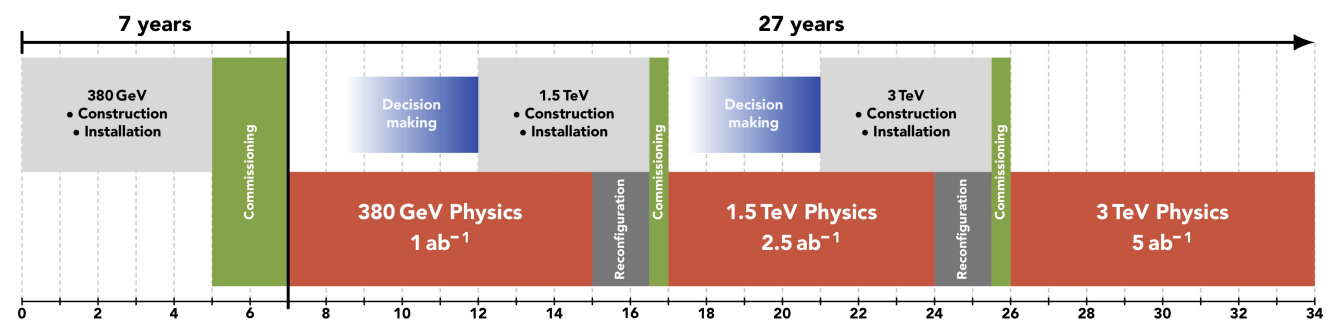

Figure 4: Proposed construction and running schedule of CLIC [8].
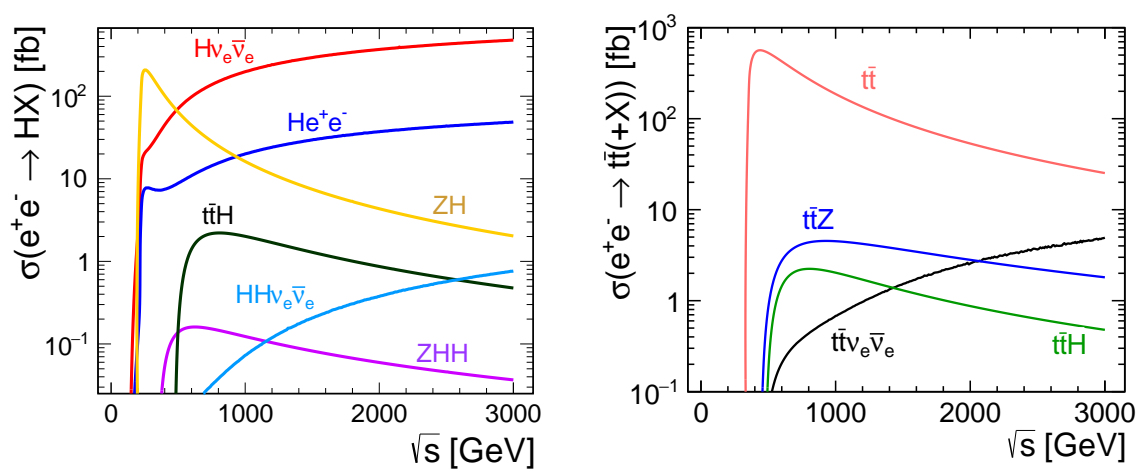

Figure 5: Production cross section as a function of centre-of-mass energy for the main Higgs boson (left) and top quark (right) production processes at $\mathrm{e}^{+} \mathrm{e}^{-}$collider [10].

at the $\mathrm{t} \overline{\mathrm{t}}$ production threshold. The second and third construction stages at $1.5 \mathrm{TeV}$ and $3 \mathrm{TeV}$, with expected integrated luminosities of $2.5 \mathrm{ab}^{-1}$ and $5 \mathrm{ab}^{-1}$, will focus on the searches for BSM phenomena. However, they will also open possibilities for additional Higgs and top-quark measurements, such as the double Higgs boson production or direct determination of the top Yukawa coupling, see Fig 5. Only the electron beam polarisation is currently included in the CLIC baseline design. The novel acceleration technology of CLIC required substantial fundamental research. Using the CLIC Test Facility at CERN [11], which ended operation in December 2016, all key elements of the design were verified.

\subsection{Comparison of $\mathrm{e}^{+} \mathrm{e}^{-}$collider projects}

Table 1 shows a short comparison of selected collider parameters of ILC and CLIC. Both projects have a similar time span. Thanks to higher expected integrated luminosity, cleaner environment (thanks to large bunch spacing and size parameters) and positron beam polarisation, ILC is expected to give higher precision at low beam energies, in particular for Higgs physics (see next section). On the other hand, the initial stage energy of CLIC was optimised for both Higgs boson and top-quark studies and the novel acceleration technology opens prospects for going beyond the $1 \mathrm{TeV}$ scale in the subsequent energy stages. With different choices of energy stages, running scenarios of ILC and CLIC are complementary and can give independent constraints on the Standard Model and different BSM scenarios. Still, one has to note that the running scenarios will most likely be revised in the future, depending on new results from HL-LHC and other experiments. 


\begin{tabular}{|l|c|c|}
\hline & ILC & CLIC \\
\hline Technology & cold & warm \\
Acc. gradient & $35 \mathrm{MV} / \mathrm{m}$ & $72 / 100 \mathrm{MV} / \mathrm{m}$ \\
Initial energy & $250 \mathrm{GeV}$ & $380 \mathrm{GeV}$ \\
Final energy & $1 \mathrm{TeV}$ & $3 \mathrm{TeV}$ \\
Bunch spacing & $300 \mathrm{~ns}$ & $0.5 \mathrm{~ns}$ \\
Polarisation & $\mathrm{e}^{-} / \mathrm{e}^{+}$ & $\mathrm{e}^{-}$ \\
Project timeline & 31 years $^{-}$ & 27 years \\
Total luminosity & $14.2 \mathrm{ab}^{-1}$ & $8.5 \mathrm{ab}^{-1}$ \\
\hline
\end{tabular}

Table 1: Comparison of ILC and CLIC projects

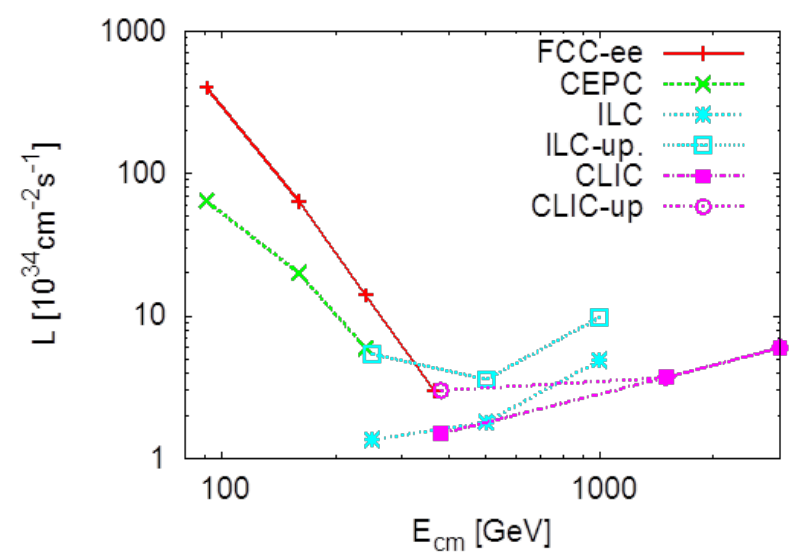

Figure 6: The instantaneous luminosities estimated for linear and circular $\mathrm{e}^{+} \mathrm{e}^{-}$collider projects as a function of the centre-of-mass energy [12]. Two IPs are assumed for the circular colliders FCC-ee and CEPC.

The instantaneous luminosities expected for future linear and circular $\mathrm{e}^{+} \mathrm{e}^{-}$collider projects as a function of their centre-of-mass energies are presented in Fig. 6. The luminosity expected at circular colliders decreases very rapidly with increasing energy and it becomes comparable to that of the linear colliders around the $t \bar{t}$ threshold. It is clear that there is no other option for $\mathrm{e}^{+} \mathrm{e}^{-}$collider at scales of $500 \mathrm{GeV}$ or above but the linear collider. There is also a significant difference in the possible upgrade path of the two options. While linear colliders can be upgraded to higher energies by extending the tunnel, increasing the acceleration gradient or implementing new acceleration technologies, a significant energy upgrade is not possible for circular colliders and one can only consider converting them into hadron machines after the $\mathrm{e}^{+} \mathrm{e}^{-}$physics program is completed.

\section{Experiments}

\subsection{Particle Flow concept}

Jet energy resolution is crucial for precision physics and background rejection. Due to large fluctuations in the hadronic cascade development, jet energy resolution of calorimeters is limited. 

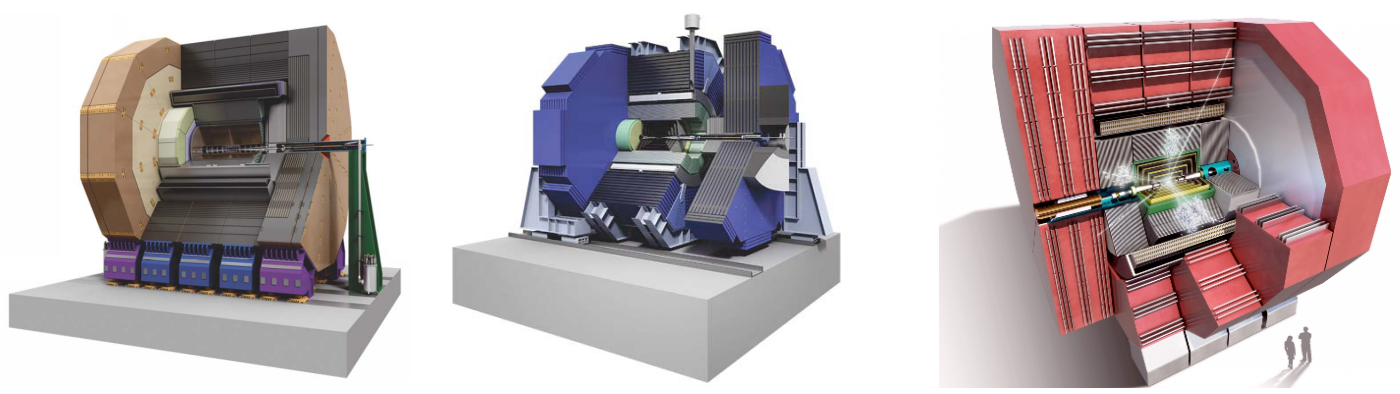

Figure 7: Detector concepts for the future linear $\mathrm{e}^{+} \mathrm{e}^{-}$colliders (from left): ILD, SiD and CLICdet.

However, significant improvement of the measurement is possible, if we are able to reconstruct and identify single particles within jets. The best possible jet energy estimate is then obtained by combining calorimeter measurements for neutral particles with much more precise track momentum measurements for the charged ones. This approach, referred to as Particle Flow Calorimetry [13], was assumed in the design of the ILC and CLIC detectors. Detector designs which were used for the physics potential studies presented in this contribution are shown in Fig. 7. Two detector concepts, ILD and SiD, have been developed for ILC [14, 15]. A dedicated detector model, CLICdet, was optimised for full exploitation of the CLIC physics potential from $380 \mathrm{GeV}$ to $3 \mathrm{TeV}$ [16].

\subsection{Detector Requirements and Performance}

Single particle reconstruction and identification is crucial for the Particle Flow approach. It is provided by very high calorimeter granularity, which allows efficient matching of calorimeter clusters with the charged particle tracks and separation of particle clusters inside the detector. For the best energy estimate of charged particles, precise momentum measurement in the wide angular and energy range is required. Figure 8 shows the expected resolution in $1 / p_{T}$ as a function of the track momentum and angle, for single muon events. For high momentum tracks produced at large angles, simulation studies indicate that a resolution of $\sigma_{1 / p_{T}} \sim 2 \cdot 10^{-5} \mathrm{GeV}^{-1}$ can be obtained. Expected jet energy resolution based on particle flow reconstruction is presented in Fig. 9, as a function of the jet emission angle for different jet energies. For high energy jets, resolution of $\sigma_{E} / E=3-4 \%$ can be obtained.

With a high precision pixel vertex detector placed very close to the beam line, precise interaction point determination and very efficient flavour tagging is possible. With the impact parameter resolution for high momentum tracks of $\sigma_{d}<5 \mu \mathrm{m}$, bottom and charm quarks can be tagged with high purity. Figure 10 compares the expected b-tagging misidentification rates for the ILD and CLICdet detectors. For quark pair production (including e.g. Higgs boson decays) light-quark background suppression by a factor $\sim 10^{-4}$ can be obtained for a $b \bar{b}$ event selection efficiency of $50 \%$. Finally, for very good detector hermeticity and efficient suppression of backgrounds to processes with missing energy, instrumentation extending down to a minimum angle of $\theta_{\min } \sim 5 \mathrm{mrad}$ is planned. 

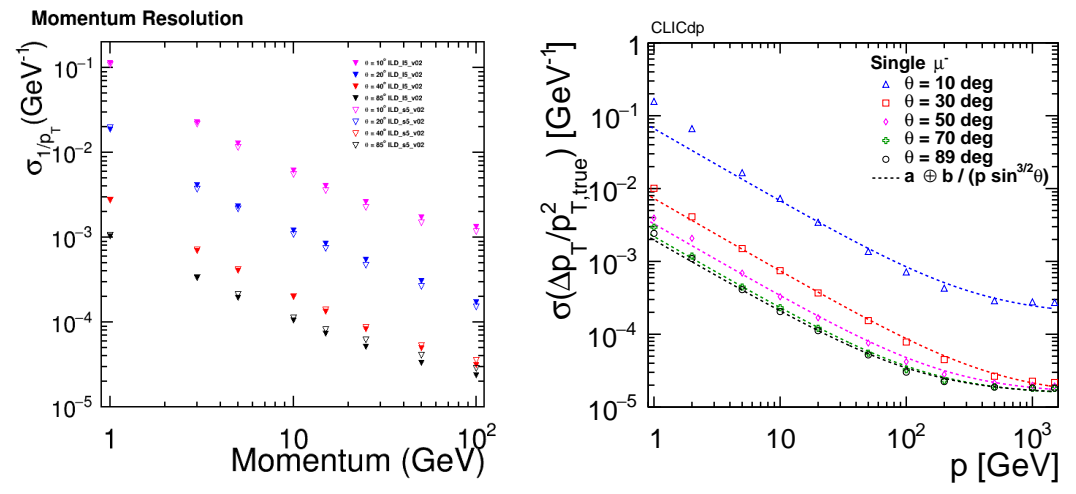

Figure 8: Expected resolution in $1 / p_{T}$ as a function of the track momentum for single muon events, for ILD [15] (left) and CLICdet [16] (right) detector concepts.
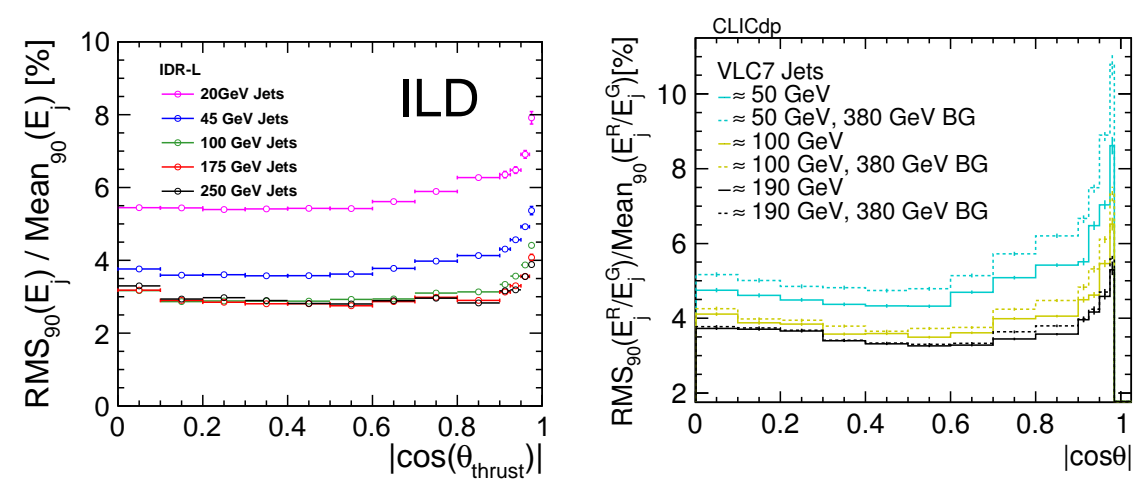

Figure 9: Particle flow performance, measured as the energy resolution for different jet energies as a function of $\cos (\theta)$, for ILD [15] (left) and CLICdet [16] (right) detector concepts. The resolution is defined as the rms of the distribution truncated so that $90 \%$ of the total jet energy is contained inside the distribution.
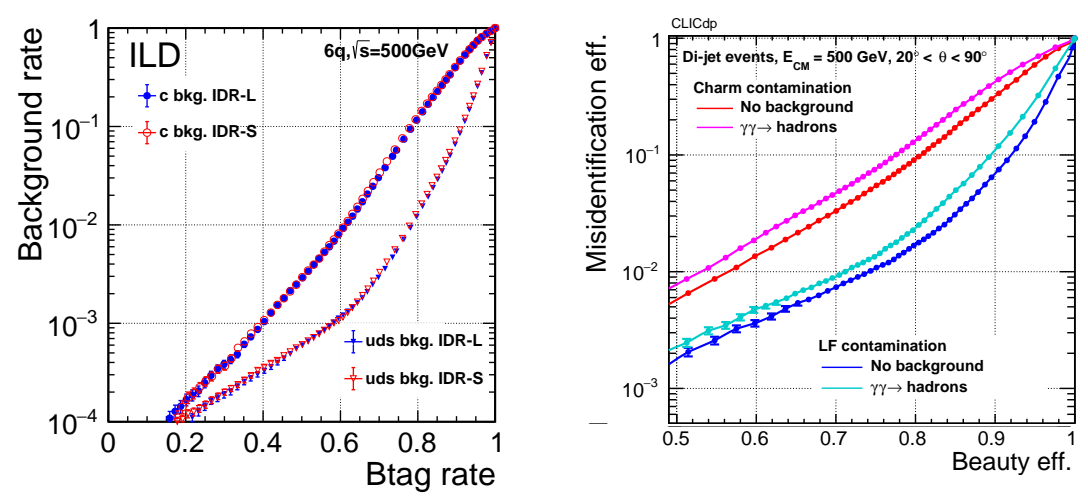

Figure 10: Misidentification rates as a function of the b-tagging efficiency for c-quark and light flavour (u,d, s) quark jets, expected for ILD [15] (left) and CLICdet [16] (right) detector concepts. 


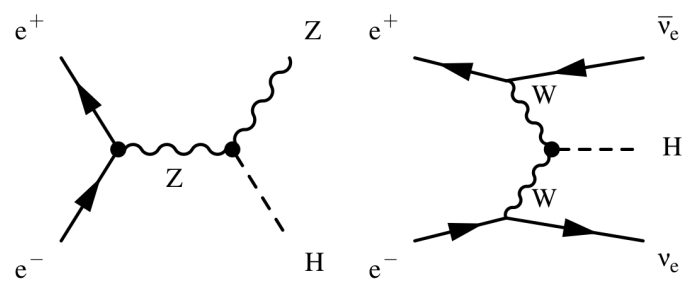

Figure 11: Leading-order Feynman diagrams of the highest cross section Higgs production processes at CLIC: Higgsstrahlung (left) and WW-fusion (right).

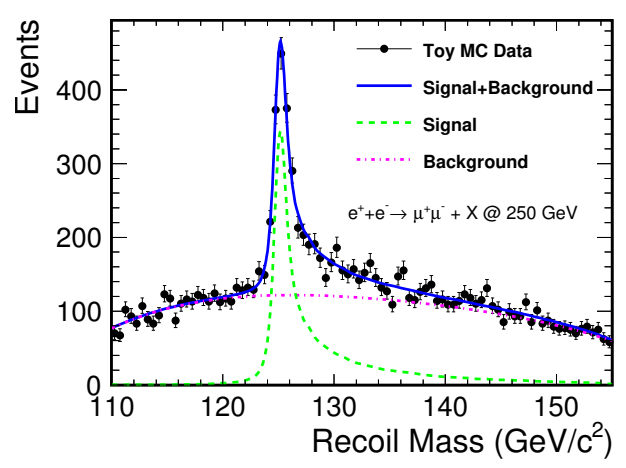

Figure 12: The recoil mass spectrum against $\mathrm{Z} \rightarrow \mu^{+} \mu^{-}$for $\mathrm{ZH}$ signal and SM background, for ILC running at $\sqrt{s}=250 \mathrm{GeV}[17]$.

\section{Higgs physics}

\subsection{Production processes}

Two processes dominate the Higgs boson production at $\mathrm{e}^{+} \mathrm{e}^{-}$collisions, see Fig. 11. For centre-of-mass energies up to about $450 \mathrm{GeV}$, the Higgs-strahlung process, Higgs boson production together with $\mathrm{Z}$ boson, dominates, see Fig. 5. Measurement of the Higgs boson production in the WW-fusion process becomes increasingly important at higher energy stages, however already at the initial ILC and CLIC stages it is helpful for model-independent extraction of the Higgs boson couplings.

\subsection{Event reconstruction}

In the ZH production channel we can use "Z-tagging" approach for unbiased selection of Higgs production events, i.e. making no assumptions about the Higgs boson decays. Figure 12 shows the recoil mass distribution for Higgsstrahlung events and SM background processes, for a Z boson decaying into a muon pair at the initial ILC stage [17]. We observe a sharp peak in the recoil mass distribution, which allows model independent tagging of the Higgs boson production events. In the clean linear collider environment, unambiguous separation of different decay channels is then possible. In particular, Higgs boson decays into two hadronic jets can be easily separated into $b \bar{b}, c \bar{c}$ and gg decays, as shown in Fig. 13 [18]. This demonstrates clear prospects for direct measurement of $\mathrm{BR}(\mathrm{H} \rightarrow \mathrm{c} \overline{\mathrm{c}})$ and $\mathrm{BR}(\mathrm{H} \rightarrow \mathrm{gg})$ at both ILC and CLIC. 


\subsection{Higgs couplings}

Future linear colliders guarantee sub-percent level precision for Higgs coupling measurements even at the first energy stages. The precision of Higgs boson coupling measurements at subsequent ILC [5] and CLIC [19, 20] stages is summarised in Figs. 14 and 15, for model-dependent and model-independent analysis approaches, respectively. For the model-dependent coupling fit, where it is assumed that there are no non-Standard-Model Higgs decays nor anomalous couplings, the expected precision is up to an order of magnitude better than expected at HL-LHC. Two approaches to the model-independent coupling determination are possible: scaling factors for the SM Higgs boson couplings and its total width can be fitted to the Higgs boson measurements (so called $\kappa$-framework) [20] or the global fit of all precision electroweak measurements can be performed in the framework of the Standard Model effective field theory (SMEFT) [21, 5]. Both approaches result in increased coupling uncertainties, compared to model-dependent fit, but can also be used to constrain a wider range of BSM scenarios.

The Standard Model gives exact predictions on the Higgs boson couplings to all other SM particles. Deviations from these predictions are expected in most BSM scenarios, in particular in those with extended Higgs sector. The precision of the linear $\mathrm{e}^{+} \mathrm{e}^{-}$colliders should allow discrimination between the SM expectations and other models of "new physics" from the global analysis of the Higgs boson couplings. This is illustrated in Fig. 16 for the ILC running at $250 \mathrm{GeV}$ and for the combined $250 \mathrm{GeV}$ and $500 \mathrm{GeV}$ data [22]. Considered is a set of benchmark BSM models which are expected to escape the direct search at HL-LHC. Significant (above 5 $\sigma$ ) discrimination between most scenarios will already be possible at $250 \mathrm{GeV}$ ILC. After the full ILC programme, all BSM scenarios considered in the study can be identified at $\geq 5 \sigma$ level.

\subsection{Invisible decays}

As already mentioned above, Higgs boson production in the Higgsstrahlung process allows a model independent determination of the Higgs boson properties at future linear colliders. Large samples of events can be selected when the hadronic decay channel of the $Z$ boson is considered. Events with mono- $Z$ production, and no other activity in the detector, can be considered as candidate events for the invisible Higgs boson decays, if the recoil mass reconstructed from energymomentum conservation is consistent with the Higgs boson mass. Figure 17 shows the expected recoil mass distribution for events with two jets in the final state, for ILC running at $\sqrt{s}=250 \mathrm{GeV}$ [5]. The main SM background processes in this analysis are the production of $\mathrm{ZZ}$ and $\mathrm{W}^{+} \mathrm{W}^{-}$ pairs, as well as single $\mathrm{Z}$ production via the WW fusion, $\mathrm{e}^{+} \mathrm{e}^{-} \rightarrow \mathrm{v}_{\mathrm{e}} \overline{\mathrm{v}}_{\mathrm{e}} \mathrm{Z}$. Also indicated is the expected contribution of invisible Higgs boson decays, assuming branching ratio $\mathrm{BR}(\mathrm{H} \rightarrow$ inv $)=$ $10 \%$. With $2 \mathrm{ab}^{-1}$ collected at $250 \mathrm{GeV}$ ILC the expected 95\% C.L. limit on invisible decays of the $125 \mathrm{GeV}$ Higgs boson is $0.23 \%$ [23].

\subsection{Higgs boson self-coupling measurement}

Measurement of the trilinear Higgs boson coupling, which provides a direct probe of the shape of the Higgs potential, is a crucial test of the Standard Model and of the electroweak symmetry breaking mechanism in general. The coupling can be constrained using indirect measurements, as the triple Higgs coupling does affect single Higgs boson production via radiative corrections. 

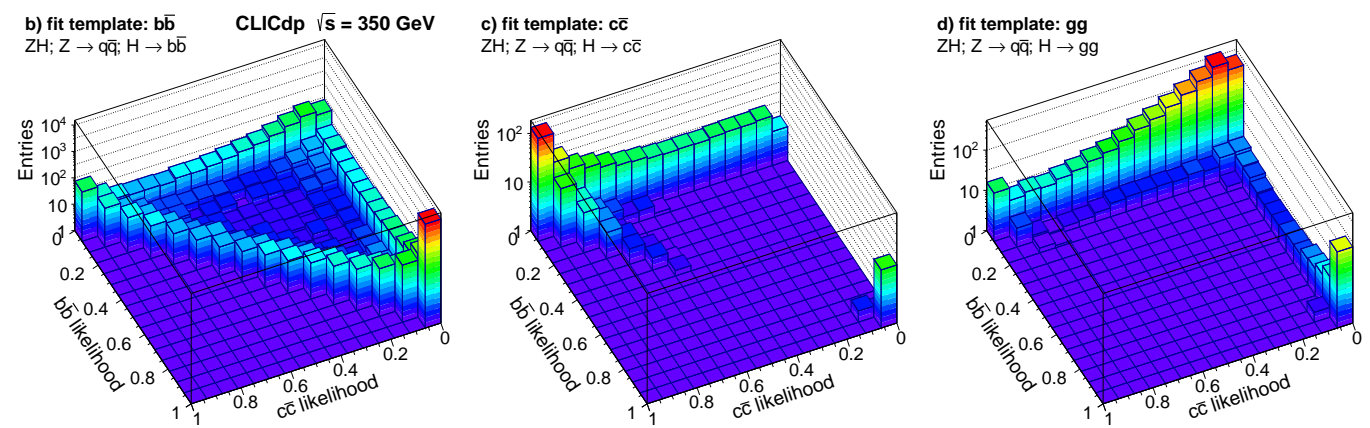

Figure 13: Performance of the flavour tagging in Higgs boson decays: $b \bar{b}$ likelihood versus c $\bar{c}$ likelihood distributions for $\mathrm{e}^{+} \mathrm{e}^{-} \rightarrow \mathrm{ZH}$ events at $\sqrt{s}=350 \mathrm{GeV}$, for the different event classes: $\mathrm{H} \rightarrow \mathrm{b} \overline{\mathrm{b}}$ (left), $\mathrm{H} \rightarrow \mathrm{c} \overline{\mathrm{c}}$ (center) and $\mathrm{H} \rightarrow \mathrm{gg}$ (right) [18].
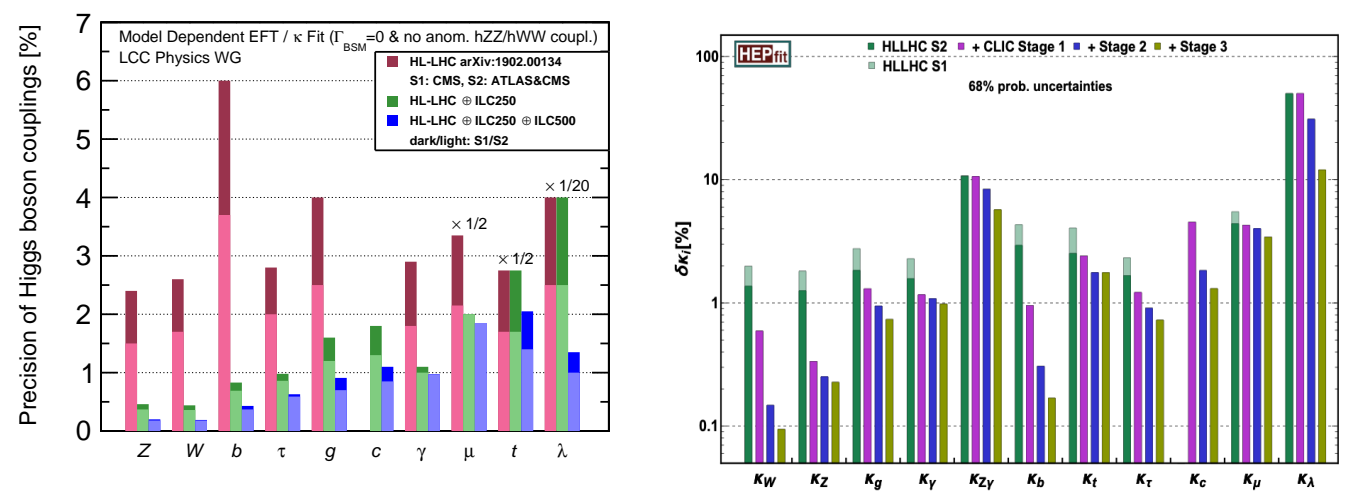

Figure 14: Precision of the Higgs couplings determined in a model-dependent fit, estimated for ILC [5] (left) and CLIC [19] (right) running at subsequent energy stages, compared with the expectations for the HL-LHC.
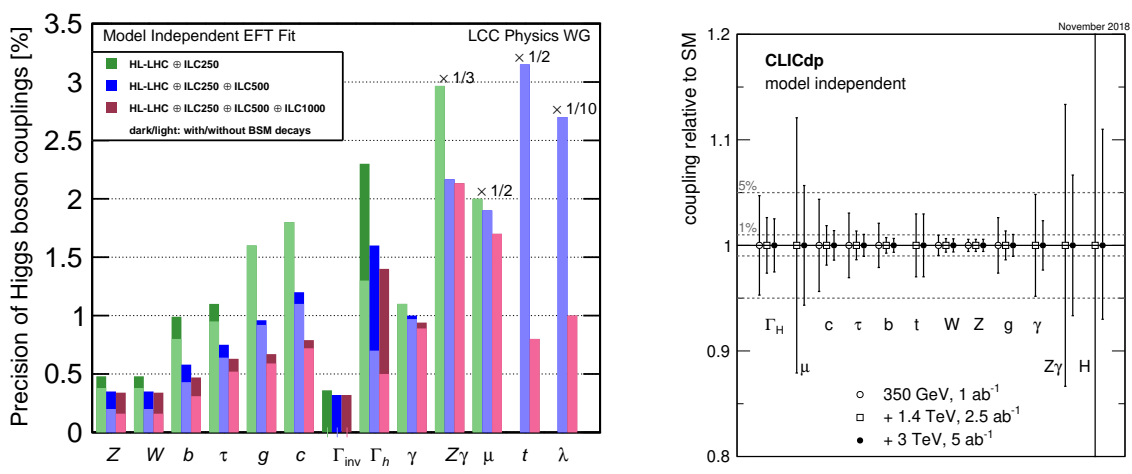

Figure 15: Precision of the model-independent Higgs coupling determination from the SMEFT analysis at the ILC [5] (left) and from the $\kappa$-framework fit at CLIC [20] (right), for subsequent energy stages. 
However, more robust determination of the triple Higgs coupling is possible by direct measurement of Higgs pair production processes involving the trilinear coupling at the tree level.

Two processes are considered for direct Higgs self-coupling measurement: double Higgs boson production in the Higgsstrahlung-like process, $\mathrm{e}^{+} \mathrm{e}^{-} \rightarrow \mathrm{ZHH}$ and double Higgs boson production in the WW-fusion process, $\mathrm{e}^{+} \mathrm{e}^{-} \rightarrow v \bar{v} \mathrm{HH}$, which becomes important only at high beam energies, see Fig. 5. Figure 18 presents the expected uncertainties in the Higgs self-coupling determination for these two processes, as a function of the centre-of-mass energy [5]. ILC running at $500 \mathrm{GeV}$ seems to be optimal for the measurement of the trilinear Higgs boson coupling in the $\mathrm{e}^{+} \mathrm{e}^{-} \rightarrow$ ZHH channel. The dedicated study indicates that the total cross section for this process can be measured with $4 \mathrm{ab}^{-1}$ to about $17 \%$ corresponding to $27 \%$ uncertainty on the trilinear coupling value [5]. For higher centre-of-mass energies, better accuracy can be expected from the measurement of $\mathrm{e}^{+} \mathrm{e}^{-} \rightarrow v \bar{v} \mathrm{HH}$. Prospects for constraining both the trilinear Higgs self-coupling and the quartic HHWW coupling from the measurement of double Higgs boson production at CLIC were studied in [24]. Figure 19 gives the BDT response distribution expected at 3 TeV CLIC and the confidence contours at $68 \%$ and $95 \%$ C.L. for the simultaneous fit of the trilinear coupling, $\kappa_{H H H}$, and quartic coupling, $\kappa_{H H W W}$, based on CLIC measurements at $1.4 \mathrm{TeV}$ and $3 \mathrm{TeV}$. The trilinear Higgs self-coupling can be constrained to $\delta \lambda / \lambda=-7 \% /+11 \%$ (68\% C.L.) [24].

\subsection{Looking for BSM effects}

All precision measurements, including Higgs boson production and other relevant SM processes, can be combined in a more general analysis based on the effective field theory (EFT) approach. An example of such an analysis for CLIC is presented in Fig. 20 for the composite Higgs model $[19,25]$. The model is characterised by the typical composite-sector mass $m^{\star}$ and coupling $g^{\star}$. The expected CLIC discovery range $(5 \sigma)$ for Higgs compositeness is compared to expected HL-LHC $2 \sigma$ exclusion limits. CLIC running at $\mathrm{TeV}$ energies can be sensitive to the compositeness mass scales up to about $50 \mathrm{TeV}$ going far beyond the HL-LHC reach for this model.

\section{Top-quark physics}

With an expected value of the Yukawa coupling of the order of one, the precise determination of the top quark properties is crucial for the understanding of electroweak symmetry breaking and of the vacuum stability of the Standard Model. As the top quark gives large loop contributions to many precision measurements, determination of its properties is also essential for many "new physics" searches.

\subsection{Top-quark mass}

The dependence of the theoretical top pair production cross section on the centre-of-mass energy shows a clear resonance-like structure at the threshold, corresponding to a narrow $t \bar{t}$ bound state. The threshold cross section is strongly affected by the smearing due to luminosity spectra (which can be partly reduced by using dedicated running configuration) and initial state radiation (ISR), see Fig. 21 [26]. The shape of the cross section is also very sensitive to other top quark properties and model parameters: top-quark width $\Gamma_{t}$, Yukawa coupling $y_{t}$ and strong coupling constant $\alpha_{s}$. Still, the threshold scan is considered the best approach to determine the top-quark mass, $m_{t}$, 

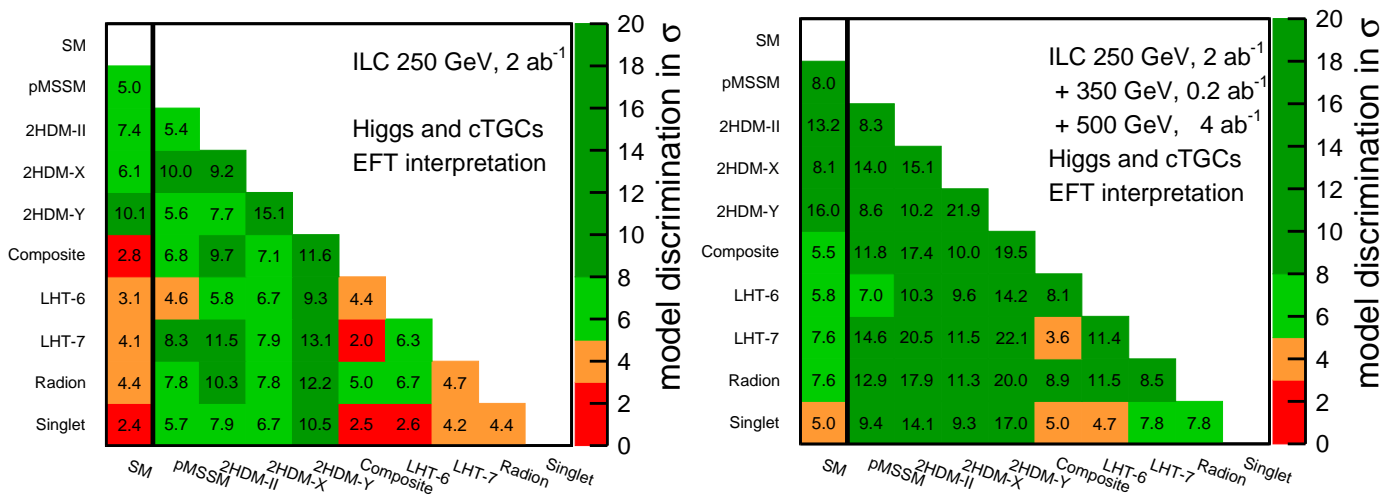

Figure 16: Expected discrimination power of the Higgs boson coupling fit at the ILC for the Standard Model and different BSM scenarios: (left) with $2 \mathrm{ab}^{-1}$ of data at $250 \mathrm{GeV}$ and (right) with $2 \mathrm{ab}^{-1}$ of data at $250 \mathrm{GeV}$ plus $4 \mathrm{ab}^{-1}$ at $500 \mathrm{GeV}$ [22].

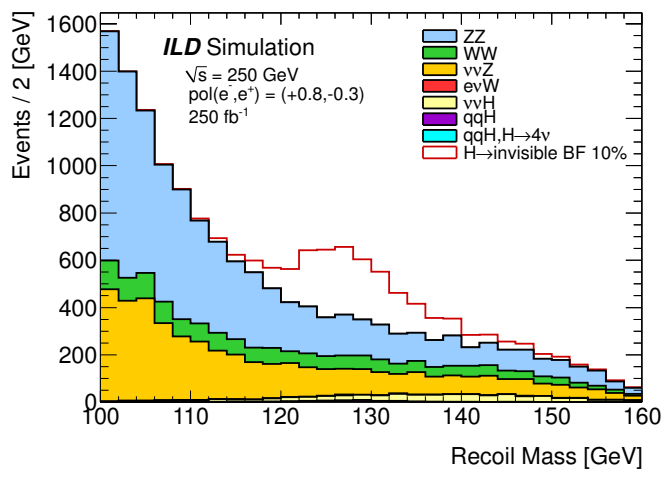

Figure 17: The recoil mass distribution for events with two jets in the final state, for ILC running at $\sqrt{s}=$ $250 \mathrm{GeV}$ and right-handed beam polarisation [5]. Open red histogram indicates the expected contribution from invisible Higgs boson decays for $\mathrm{BR}(\mathrm{H} \rightarrow \mathrm{inv})=10 \%$.
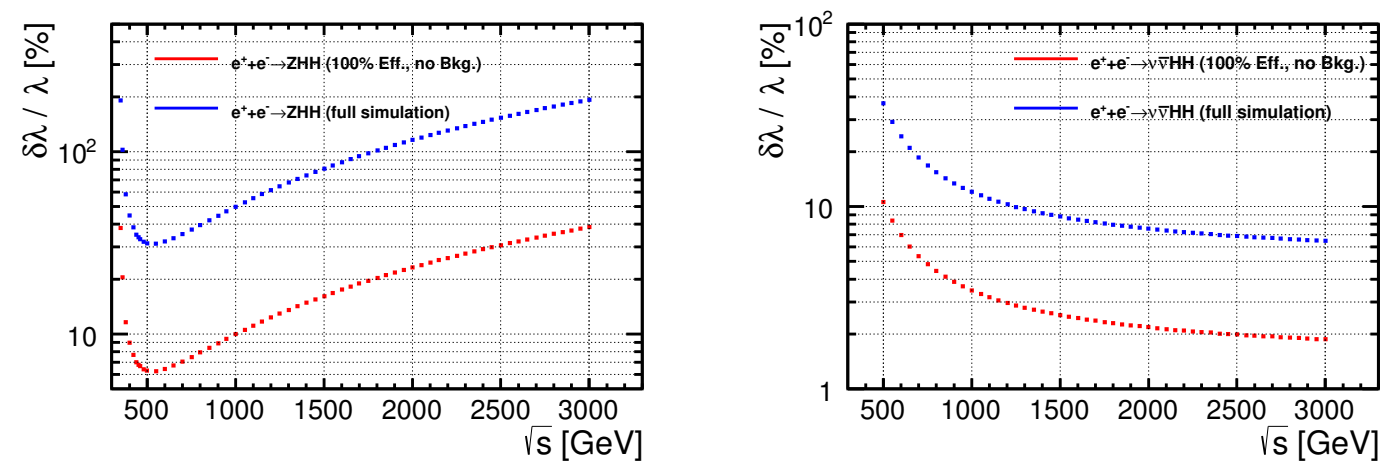

Figure 18: Estimated precision on the determination of the Higgs self-coupling $\lambda$ from the measurement of the double Higgs production: $\mathrm{e}^{+} \mathrm{e}^{-} \rightarrow \mathrm{ZHH}$ (left) and $\mathrm{e}^{+} \mathrm{e}^{-} \rightarrow v \bar{v} \mathrm{HH}$ (right), as a function of the centreof-mass energy. Same integrated luminosities of $4 \mathrm{ab}^{-1}$ is assumed at all $\sqrt{s}$ [5]. 

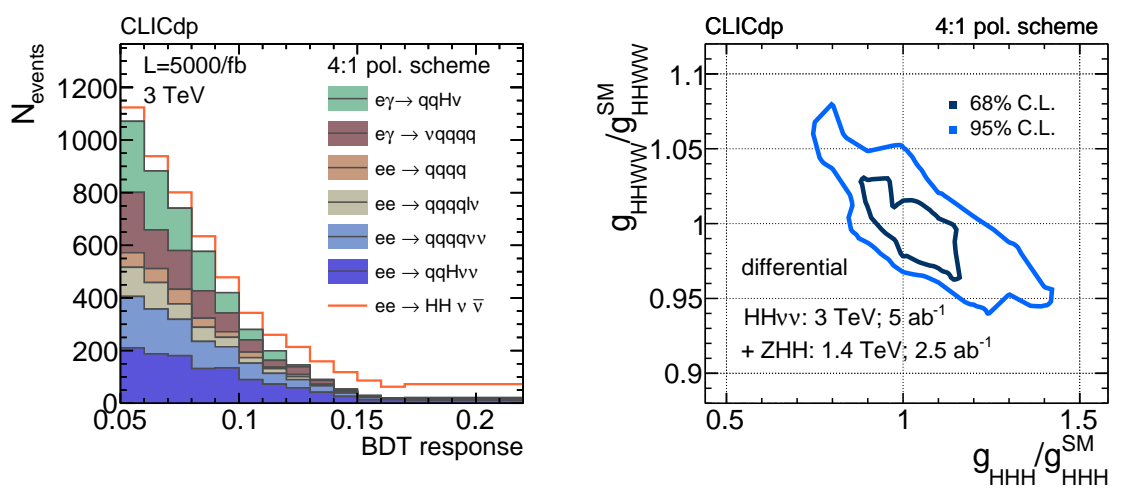

Figure 19: Left: BDT response distribution of all SM contributions stacked in the loose BDT selection at 3 TeV CLIC. Right: confidence contours at $68 \%$ and $95 \%$ C.L. for the simultaneous fit of $\kappa_{H H H}$ and $\kappa_{H H W W}$ based on CLIC measurements at $1.4 \mathrm{TeV}$ and $3 \mathrm{TeV}$ [24].

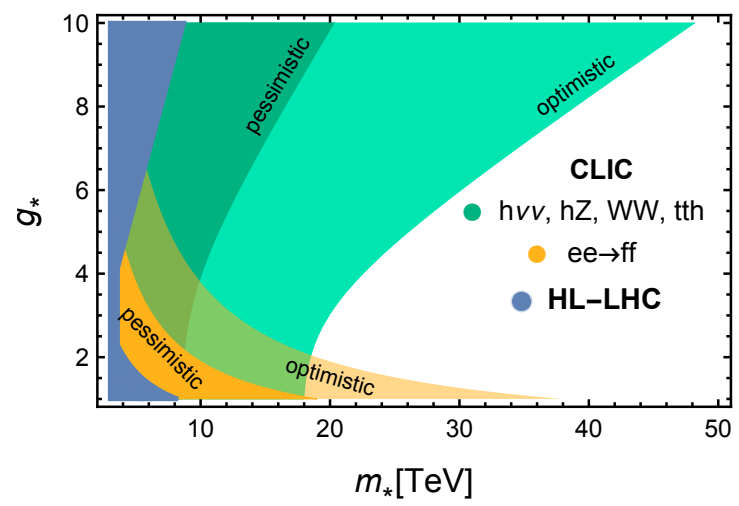

Figure 20: Expected $5 \sigma$ discovery contours for Higgs compositeness in the $\left(m^{\star}, g^{\star}\right)$ plane, overlaid with the $2 \sigma$ projected exclusions from HL-LHC [25].

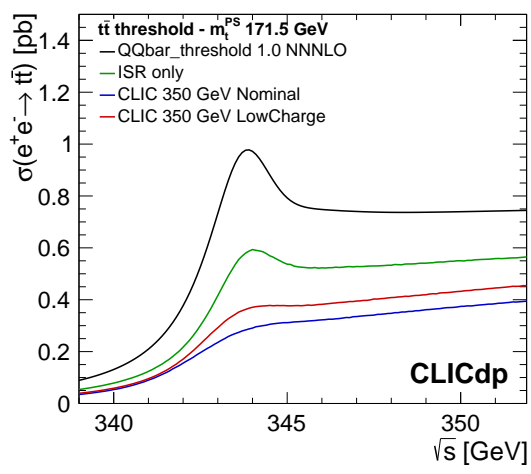

Figure 21: The impact of the initial state radiation (ISR) and the luminosity spectra on the top-quark pair production cross section at the threshold. The blue and red curves show the observable cross section for the nominal and the "reduced charge" luminosity spectra for CLIC, respectively [26]. 

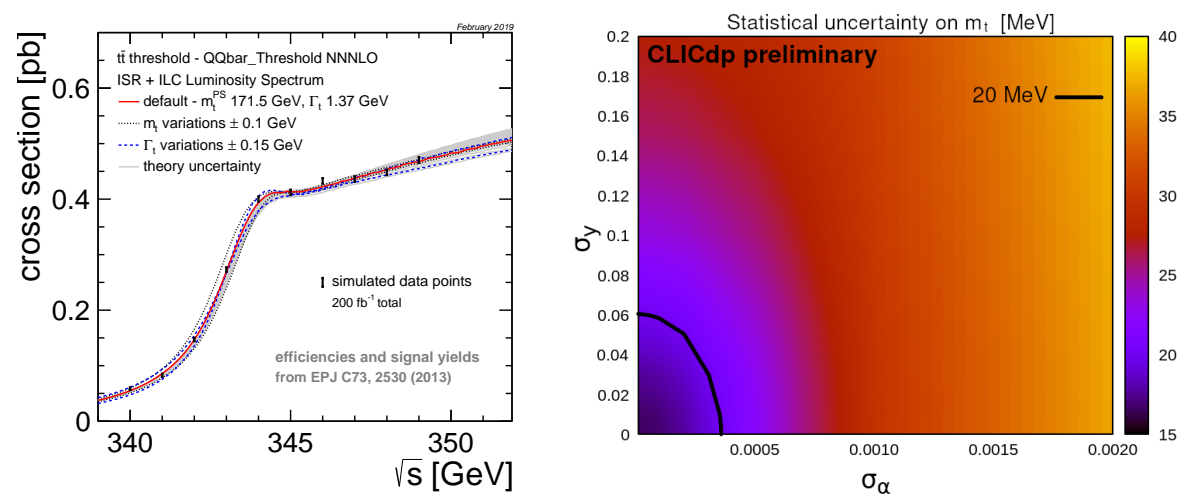

Figure 22: Left: reference scenario for the top-quark threshold scan considered for the ILC [5]. Right: expected statistical uncertainty on the top-quark mass from the fit of four model parameters $\left(m_{t}, \Gamma_{t}, y_{t}\right.$ and $\alpha_{s}$ ) as a function of the uncertainties on the strong coupling constant and the Yukawa coupling values from earlier measurements, assuming total normalisation uncertainty (data + theory) of $0.1 \%$ [27].
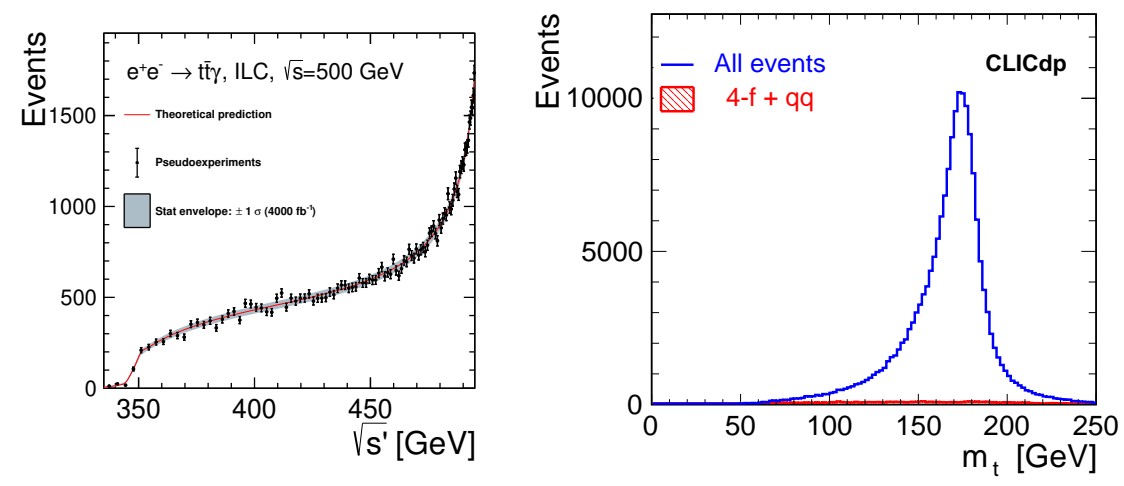

Figure 23: Expected distributions of (left) the effective $t \bar{t}$ centre-of-mass energy for radiative events at $500 \mathrm{GeV}$ ILC [28] and (right) distribution of the top-quark mass reconstructed for the semi-leptonic samples of top-quark pair-production events at $380 \mathrm{GeV}$ CLIC [26].

with highest possible precision. The other advantage the threshold scan is that the extracted topquark mass parameter is well defined from the theoretical point of view.

Baseline threshold scan scenario assumes running at 10 equidistant energy points taking 10$20 \mathrm{fb}^{-1}$ of data for each value of $\sqrt{s}$. Such a scenario is indicated in Fig. 22 (left) [5]. A statistical uncertainty of about $20 \mathrm{MeV}$ is expected from a mass and width fit and the total systematic uncertainty should be controlled to the level of $50 \mathrm{MeV}[5,26]$. However, to reach this level of statistical uncertainty, the strong coupling constant and the top-quark Yukawa coupling need to be constrained from independent measurements, see Fig. 22 (right) [27]. Longitudinal beam polarisation has not been considered in these studies.

The top-quark mass can also be extracted from measurements at higher centre-of-mass energies. One of the options is the threshold cross section determination from radiative events $\mathrm{e}^{+} \mathrm{e}^{-} \rightarrow \mathrm{t} \overline{\mathrm{t}} \gamma$, see Fig. 23 (left). Measurement of a threshold in the reconstructed $\mathrm{t} \overline{\mathrm{t}}$ invariant mass should allow for top-quark mass extraction with a statistical precision of the order of $90 \mathrm{MeV}$ for CLIC running at $380 \mathrm{GeV}$ and $110 \mathrm{MeV}$ for ILC running at $500 \mathrm{GeV}$, for integrated luminosities of 

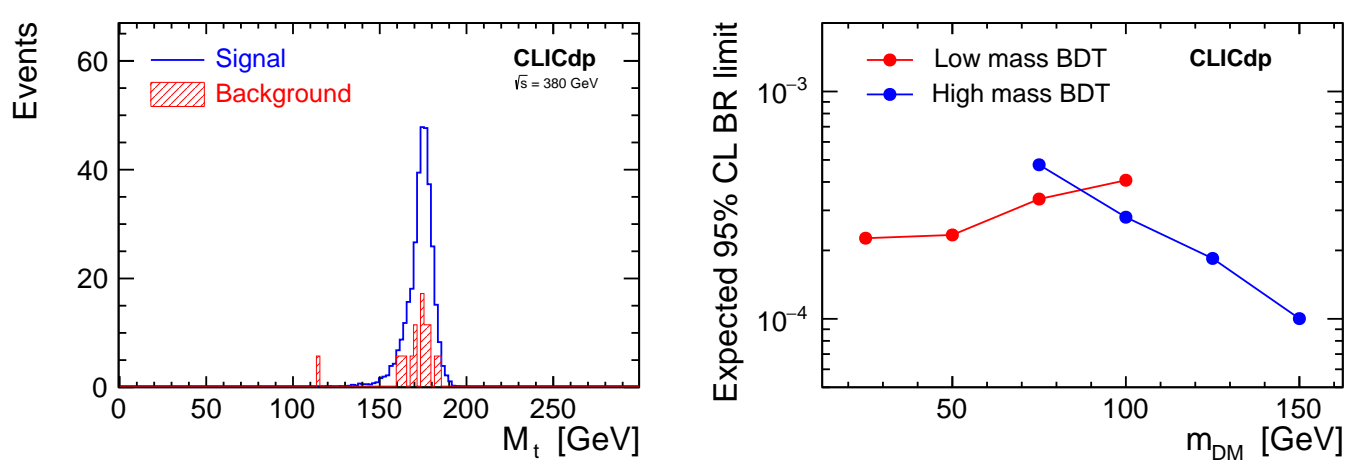

Figure 24: Left: invariant mass distribution of the top quark from the FCNC decay $\mathrm{t} \rightarrow \mathrm{c} \gamma$ reconstructed at $380 \mathrm{GeV}$ CLIC after selection based on the BDT response, normalised to $1.0 \mathrm{ab}^{-1}$ and $\mathrm{BR}(\mathrm{t} \rightarrow \mathrm{c} \gamma)=10^{-3}$ for the signal events. Right: limits at 95\% C.L. on the top quark FCNC decay $\mathrm{t} \rightarrow \mathrm{cE}$ expected for $1.0 \mathrm{ab}^{-1}$ collected at $380 \mathrm{GeV}$ CLIC [26].

$1 \mathrm{ab}^{-1}$ and $4 \mathrm{ab}^{-1}$, respectively [28]. Taking systematic effects into account, the total uncertainty of the extracted top-quark mass is $110 \mathrm{MeV}$ at the initial stage of CLIC and $150 \mathrm{MeV}$ for ILC running at $500 \mathrm{GeV}$. The top-quark mass can be also measured directly from reconstruction of hadronic top-quark decays, see Fig. 23 (right). For the initial stage of CLIC, the statistical precision of about $30 \mathrm{MeV}$ is expected for combined hadronic and semi-leptonic samples of top-quark pair-production events [26]. The measurement requires excellent control of the jet energy scale and the extracted mass is also subject to large theoretical uncertainties.

\subsection{FCNC top-quark decays}

Flavour-Changing Neutral Current (FCNC) top quark decays are very strongly suppressed in the Standard Model, with the expected branching ratios $\mathrm{BR}(\mathrm{t} \overline{\mathrm{t}} \rightarrow \mathrm{cX}) \sim 10^{-15}$ to $10^{-12}$ (X= $\gamma, g, Z, H)$. Observation of any such decay would be a direct signature for "new physics". Significant enhancement is expected in many BSM scenarios, reaching up to $10^{-2}$ for $\mathrm{BR}(\mathrm{t} \rightarrow \mathrm{cH})$ and $10^{-5}$ for $\mathrm{BR}(\mathrm{t} \rightarrow \mathrm{c} \gamma)$. Three FCNC decay channels involving charm quark have been studied for the first stage of CLIC. All channels profit from the precise final state reconstruction and high flavour tagging efficiency. Selected results are shown in Fig. 24. Limits at 95\% C.L. expected for $1 \mathrm{ab}^{-1}$ collected at $380 \mathrm{GeV}$ CLIC are [26]:

$$
\begin{aligned}
\mathrm{BR}(\mathrm{t} \rightarrow \mathrm{c} \gamma) & <2.6 \cdot 10^{-5}, \\
\mathrm{BR}(\mathrm{t} \rightarrow \mathrm{cH}) \times \mathrm{BR}(\mathrm{H} \rightarrow \mathrm{b} \overline{\mathrm{b}}) & <8.8 \cdot 10^{-5} \\
\mathrm{BR}(\mathrm{t} \rightarrow \mathrm{cE}) & <1.0-3.4 \cdot 10^{-4},
\end{aligned}
$$

where the limit for the top-quark decay involving invisible massive scalar particle, $\mathrm{t} \rightarrow \mathrm{cE}$, depends on the assumed particle mass, see Fig. 24 (right). For channels involving charm quark, limits expected at ILC and CLIC are much stronger than those estimated for HL-LHC [29, 30].

\subsection{Constraining top-quark couplings}

Measurements of the t $\bar{t}$ production cross section and angular distributions for different centreof-mass energies and beam polarisations can be used for precise determination of the top-quark 

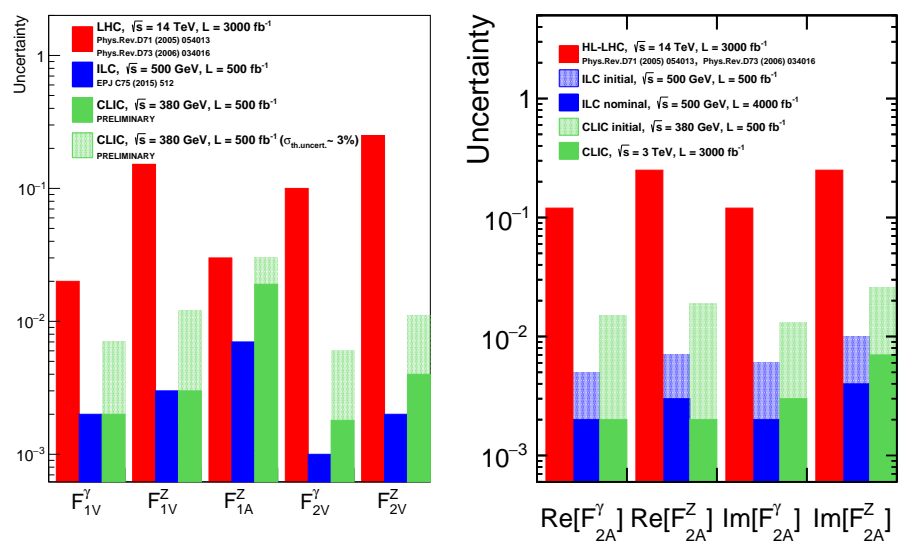

Figure 25: Comparison of the uncertainties on CP-conserving (left) and CP-violating (right) form factors in top-quark electroweak couplings expected at the HL-LHC, ILC and CLIC [31].

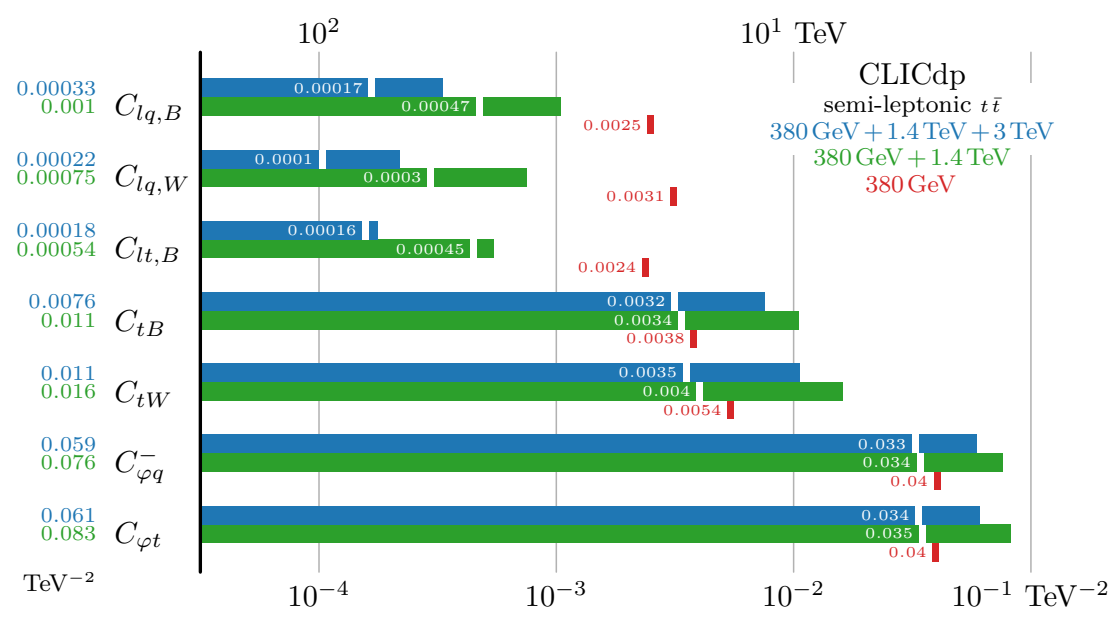

Figure 26: Summary of the global EFT analysis results using statistically optimal observables for the three CLIC energy stages. The colour bars indicate the $1 \sigma$ constraints on each of the seven Wilson coefficients. The corresponding individual operator sensitivities are shown as ticks [26].

couplings to the photon and the Z-boson, and to constrain possible BSM effects. The expected sensitivity of ILC and CLIC to electroweak couplings of the top quark is presented in Fig. 25 in terms of the uncertainties on the CP-conserving and CP-violating form factors [31].

Effects induced by heavy new physics can be also described in terms of Effective Field Theory (EFT) operators. Expected limits on the Wilson coefficients for seven EFT operators contributing to the top-quark pair production, resulting from the global EFT analysis of all measurements based on statistically optimal observables, are presented in Fig. 26. Even at the first CLIC stage, mass scales in the $10 \mathrm{TeV}$ range can be probed. For four operators, most of the sensitivity is provided by the initial $380 \mathrm{GeV}$ stage [26]. These results confirm that high energy CLIC can reach "new physics" scales in the $100 \mathrm{TeV}$ domain. 


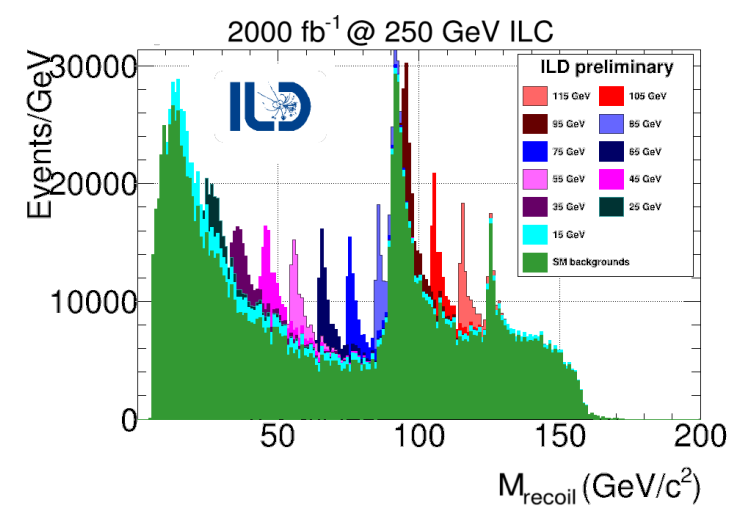

Figure 27: The recoil mass distributions for signals of new scalar production with different masses and for all SM backgrounds, after the selection cuts, for ILC running at $250 \mathrm{GeV}$ [32].

\section{BSM physics}

Strong limits on many BSM scenarios are expected from the experiments at HL-LHC. However, searches which can be performed at $\mathrm{e}^{+} \mathrm{e}^{-}$colliders are in many cases complementary. Two approaches are possible: direct BSM searches, in particular for models with weak couplings or soft signatures, which are difficult to be constrained at the LHC and indirect searches, where the sensitivity to "new physics" effects can reach very high energy scales thanks to high measurement precision and clean environment.

\subsection{Search for new scalars}

Many BSM models introduce extended Higgs sectors. If couplings of new scalars to SM particles are small, they are not excluded by the current experimental constraints, even for masses of the order of $10-100 \mathrm{GeV}$. Such a new scalar, $S^{0}$, if coupling to the SM gauge bosons, could be produced in the "Scalarstrahlung" process, $\mathrm{e}^{+} \mathrm{e}^{-} \rightarrow \mathrm{ZS}^{0}$. With very weak couplings to SM particles, invisible decays of the new scalar are expected to dominate. Such decays can be constrained by looking at events with single Z-boson production and searching for a peak in the recoil mass distribution (similar to the search for invisible Higgs boson decays presented above). An example of the reconstructed recoil mass distribution for SM background processes and new scalar production with different masses, for ILC running at $250 \mathrm{GeV}$, is presented in Fig. 27. As the recoil mass can be very precisely reconstructed for Z-boson decays into two muons, new scalar production should be clearly visible as an additional structure in the recoil mass distribution. Resulting limits on the production cross section of the new scalar, relative to the expected SM Higgs production cross section at given mass, estimated for ILC running at $250 \mathrm{GeV}$ and $500 \mathrm{GeV}$ [32] are shown in Fig. 28 (left). In the study performed for CLIC running at $380 \mathrm{GeV}$ [33], see Fig. 28 (right), hadronic decays of the Z-boson were considered and the new scalar was assumed to have only invisible decay channels. While ILC running at $250 \mathrm{GeV}$ is expected to be most sensitive to production of light new scalars below $150 \mathrm{GeV}$, running at higher centre-of-mass energy allows to extend exclusion limits to higher scalar masses. 

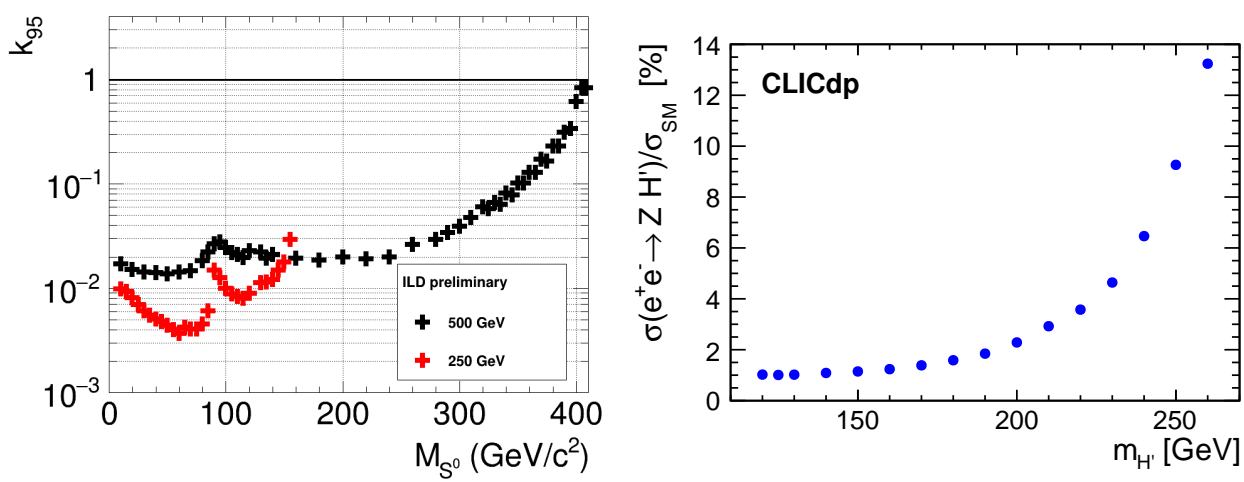

Figure 28: Expected limits on the production cross section of the new scalar, relative to the expected SM Higgs production cross section, as a function of its mass, for ILC running at $250 \mathrm{GeV}$ and $500 \mathrm{GeV}$ [32] (left) and CLIC running at $380 \mathrm{GeV}[33]$ (right).

\subsection{Inert Doublet Model}

The Inert Doublet Model is one of the simplest SM extensions providing a natural candidate for dark matter. Light IDM scenarios, with scalar masses in $\mathscr{O}(100 \mathrm{GeV})$ range are still not excluded by the current experimental and theoretical constraints [34]. Low mass IDM scenarios can be observed with high significance in the di-lepton channels already at a $\mathrm{e}^{+} \mathrm{e}^{-}$collider with $250 \mathrm{GeV}$ centre-ofmass energy [35]. The discovery reach increases for higher $\sqrt{s}$ [36] and significant improvement in the discovery reach is observed when considering the semi-leptonic final state $[37,38]$. In particular, the discovery reach for charged scalar pair-production is extended at $3 \mathrm{TeV}$ CLIC up to $m_{\mathrm{H}^{ \pm}} \sim 1 \mathrm{TeV}$. A full simulation study of the charge scalar pair-production in the semi-leptonic decay channel is ongoing. More details were given in a dedicated contribution [38].

\subsection{Dark Matter searches}

Production of Dark Matter particles in $\mathrm{e}^{+} \mathrm{e}^{-}$collisions is possible in many BSM scenarios. The unique advantage of $\mathrm{e}^{+} \mathrm{e}^{-}$colliders is that we can detect processes with invisible final states by studying the spectra of ISR photons. Expected spectra of the photon energy (relative to the beam energy: $x_{\gamma}=E_{\gamma} / E_{e}$ ) is presented in Fig. 29 for production of different SUSY particles and the SM background process $\mathrm{e}^{+} \mathrm{e}^{-} \rightarrow v \bar{v} \gamma$, for ILC running at $500 \mathrm{GeV}$ [39] and $1.5 \mathrm{TeV}$ CLIC [40]. A full simulation study performed for the ILD [41, 42] indicates that detector effects are very important and the background levels are high, see Fig. 30 (left). The $v \bar{v} \gamma$ background is strongly suppressed for the right-handed electron beam polarisation and reduction by up to a factor of 5 is expected at the ILC thanks to the positron beam polarisation [42]. Comparison of the measured photon energy spectra with SM expectations can be used to set limits on the cross section contribution from the pair-production of DM particles, as shown in Fig. 30 (right) for CLIC running at $380 \mathrm{GeV}$ [19]. Comparison of extracted mediator mass limits, for mono-photon events at future $\mathrm{e}^{+} \mathrm{e}^{-}$colliders and mono-jet events at future hadronic colliders, is presented in Fig. 31. For scalar mediator hypothesis, mediator mass limits expected at ILC and CLIC are comparable with those at FCC-hh [12]. 

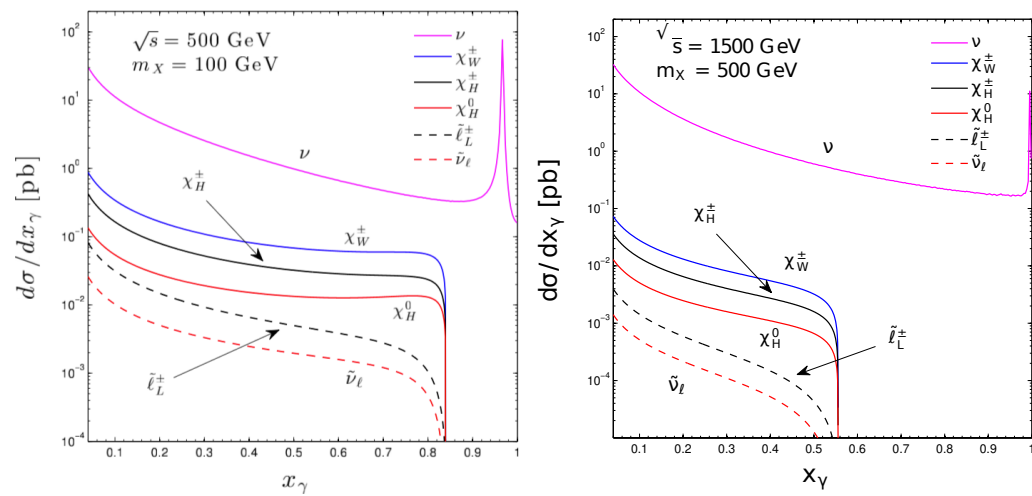

Figure 29: Unpolarized $x_{\gamma}$ distribution $\frac{d \sigma}{d x_{\gamma}}$ for different SUSY EW particles, as well as that of the background process $\mathrm{e}^{+} \mathrm{e}^{-} \rightarrow v \bar{v}$ (solid line on the top), for $m_{X}=100 \mathrm{GeV}$ at a $500 \mathrm{GeV}$ ILC [39] (left) and $m_{X}=500 \mathrm{GeV}$ at a $1500 \mathrm{GeV}$ CLIC [40] (right).
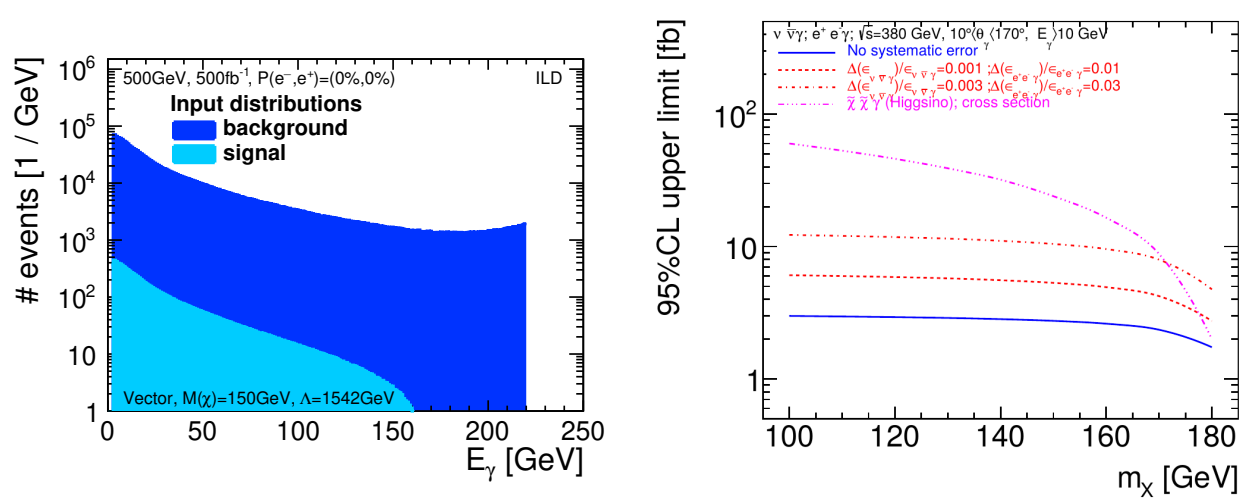

Figure 30: Left: the signal and background input histograms of the photon energy distribution, for pairproduction of DM particles with mass of $150 \mathrm{GeV}$, corresponding to the expected $95 \%$ C.L. exclusion limit for the production cross section at $500 \mathrm{GeV}$ ILC [41]. Right: upper limit (95\% C.L.) on the $\mathrm{e}^{+} \mathrm{e}^{-} \rightarrow$ $\chi \chi \gamma$ cross section at $\sqrt{s}=380 \mathrm{GeV}$ as a function of the DM particle mass, $m_{\chi}$. The limits without and with including systematic uncertainties are compared to the expected cross section for the higgsino pair production with an ISR photon [19].

\subsection{Other direct searches}

The direct (and indirect) reach of ILC and CLIC can exceed that of HL-LHC for many BSM models, in particular those with exotic scalar sector or new Higgs bosons. Shown in Fig. 32 are direct and indirect sensitivities of CLIC to new heavy scalar singlets, compared to those expected at HL-LHC [19]. Constraints at 95\% C.L. are presented in the plane of scalar mass, $m_{\phi}$, and the mixing angle of new scalar field with the SM field, $\sin 2 \gamma$. The results presented demonstrate that direct and indirect limits from CLIC are complementary and much stronger than the expected HLLHC sensitivity. High sensitivity, thanks to precision tracking and low background conditions, is also expected in searches based on the "disappearing tracks" signature. Results indicating CLIC sensitivity to charged higgsino pair production are presented in Fig. 33 [10]. 


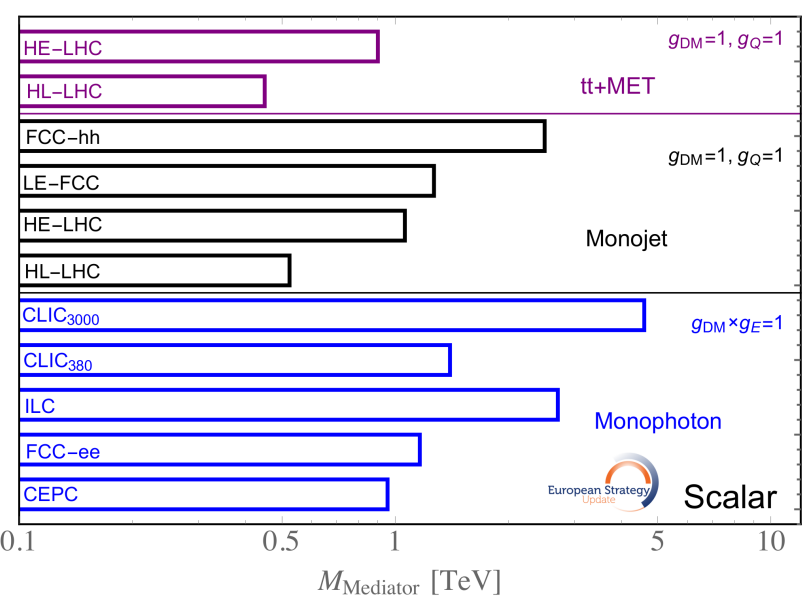

Figure 31: Summary of $2 \sigma$ sensitivity to scalar mediator mass at future colliders for simplified models with a DM mass of $M_{D M}=1 \mathrm{GeV}$ and for the couplings shown in the figure [12].

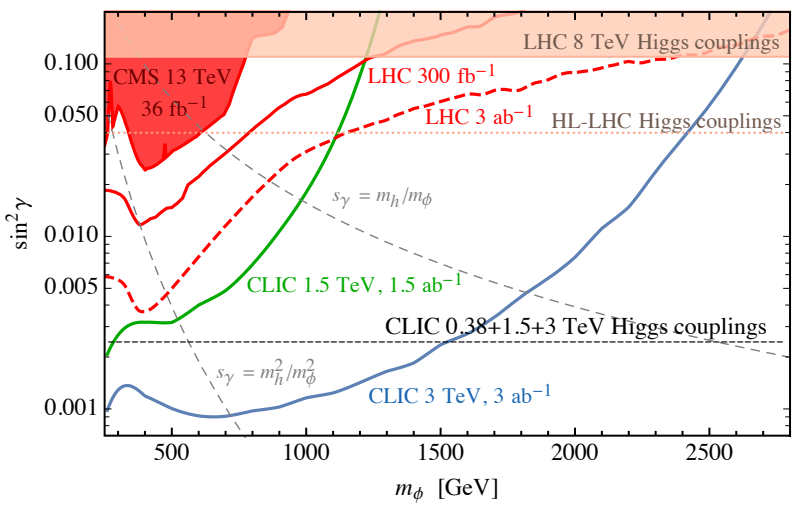

Figure 32: New scalar singlet constraints at 95\% C.L. in the plane $\left(m_{\phi}, \sin 2 \gamma\right)$. Direct and indirect limits estimated for CLIC are compared to those expected at HL-LHC. The shaded regions are the present constraints from LHC. From [19].

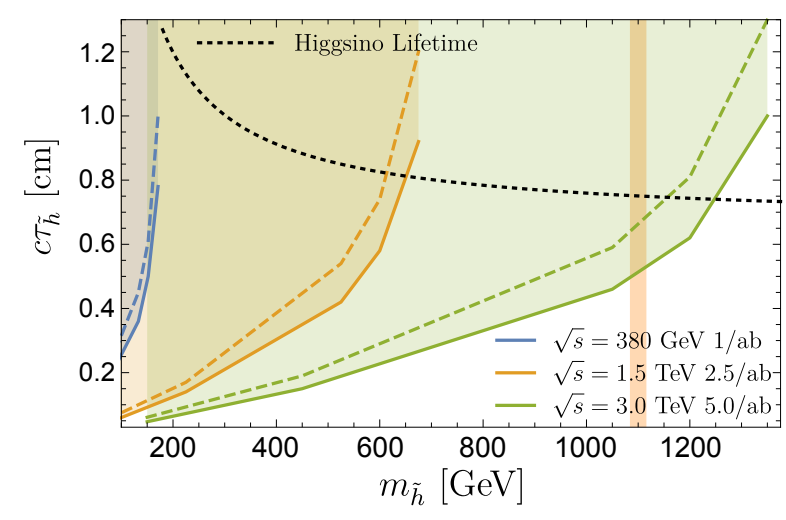

Figure 33: Contours in the lifetime-mass plane for $\mathrm{N}=3$ (solid) and $\mathrm{N}=30$ (dashed) higgsino events in the detector acceptance range at the three stages of CLIC [10]. The black dashed line indicates the lifetime of the pure Higgsino state of a given mass expected for the scenario considered in [19]. 


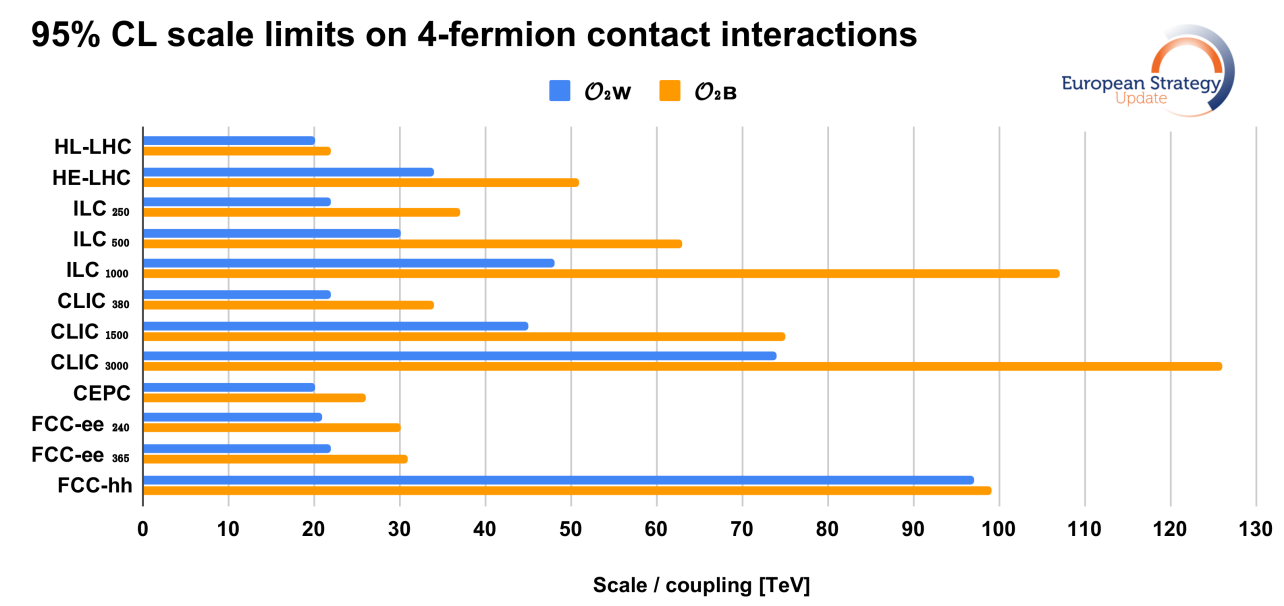

95\% CL scale limits on 2-fermion 2-boson contact interactions

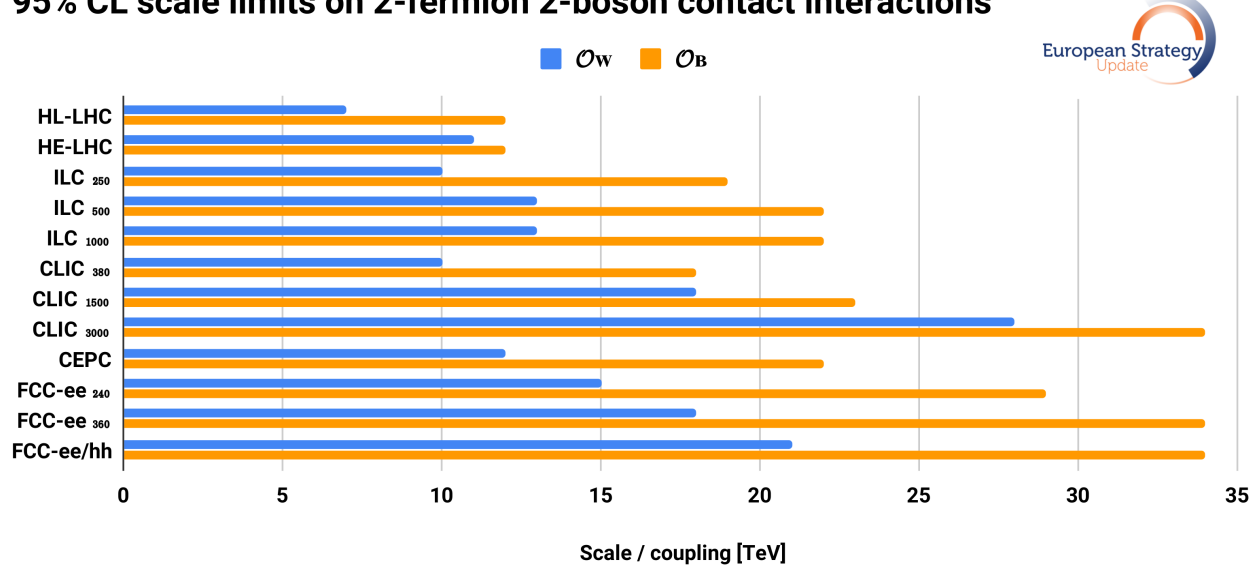

Figure 34: Exclusion reach of different colliders on: (upper plot) four-fermion contact interactions from the operators $\mathscr{O}_{2 W}$ and $\mathscr{O}_{2 B}$, and (bottom plot) two-fermion/two-boson contact interactions from the operators $\mathscr{O}_{W}$ and $\mathscr{O}_{B}[12]$.

\subsection{EFT analysis}

All collider measurements can be combined in a global EFT analysis which gives the most general constraints on the possible "new physics" effects at high energy scales. A summary of the sensitivity expected at different future colliders is presented in Fig. 34, for 4-fermion contact interactions and 2-fermion-2-boson contact interaction operators [12]. The ILC and CLIC sensitivities exceed that of HL-LHC even at their first running stages. For 4-fermion contact interactions limits reaching $100 \mathrm{TeV}$ scales, can only be obtained at (upgraded) $1 \mathrm{TeV}$ ILC, CLIC running at $3 \mathrm{TeV}$ or FCC-hh.

\section{Conclusions}

Presented in this contribution is my personal selection of results demonstrating the physics potential of ILC and CLIC. High Energy linear $\mathrm{e}^{+} \mathrm{e}^{-}$colliders offer a rich and diverse research programme: precise determination of Higgs couplings; precise determination of top-quark mass and other properties; stringent constraints on many BSM scenarios from indirect searches reaching 
mass scales up to $100 \mathrm{TeV}$ and prospects for direct observation of new physics in many scenarios. Because of the different energy choices and running scenarios, the two projects, ILC and CLIC, are to a large extent complementary. From the pure physics point of view, in would be most advantageous, if both colliders are built.

\section{Acknowledgements}

I would like to thank ILD, SiD and CLICdp groups for providing results presented in this contribution, as well as the group members for many useful comments and suggestions. This contribution was supported by the National Science Centre, Poland, the OPUS project under contract UMO-2017/25/B/ST2/00496 (2018-2021). Thanks are due to the LCC generator working group and the ILD software working group for providing the simulation and reconstruction tools and producing the Monte Carlo samples. Presented studies benefited also from computing services provided by the ILC Virtual Organization, supported by the national resource providers of the EGI Federation and the Open Science GRID.

\section{References}

[1] ILC inputs to the European Strategy for Particle Physics Update: https://ilchome.web.cern.ch/content/ilc-european-strategy-document

[2] CLIC input to the European Strategy for Particle Physics Update: https://clic.cern/european-strategy

[3] C. Adolphsen et al., The International Linear Collider Technical Design Report - Volume 3.II: Accelerator Baseline Design, arXiv:1306.6328 [physics.acc-ph].

[4] L. Evans et al., The International Linear Collider Machine Staging Report 2017, arXiv:1711.00568 [physics.acc-ph].

[5] P. Bambade et al., The International Linear Collider: A Global Project, arXiv:1903.01629 [hep-ex].

[6] T. Barklow et al., ILC Operating Scenarios, arXiv:1506.07830 [hep-ex].

[7] M. Aicheler et al., A Multi-TeV Linear Collider Based on CLIC Technology : CLIC Conceptual Design Report, CERN-2012-007.

[8] M. Aicheler et al., The Compact Linear Collider (CLIC) - Project Implementation Plan, CERN Yellow Rep. Monogr. Vol. 4 (2018), arXiv:1903.08655 [physics.acc-ph].

[9] Compact Linear Collider Project, CLIC accelerator schematic diagrams, OPEN-PHO-ACCEL-2019-002, https://cds.cern.ch/record/2655160

[10] P. N. Burrows et al., The Compact Linear Collider (CLIC) - 2018 Summary Report, CERN Yellow Rep. Monogr. Vol. 2 (2018), arXiv:1812.06018 [physics.acc-ph].

[11] R. Corsini, Final Results From the Clic Test Facility (CTF3), in Proc. IPAC2017, https://doi.org/10.18429/JACoW-IPAC2017-TUZB1, 2017.

[12] R.K. Ellis et al., Physics Briefing Book : Input for the European Strategy for Particle Physics Update 2020, arXiv:1910.11775 [hep-ex].

[13] M. A. Thomson, Particle Flow Calorimetry and the PandoraPFA Algorithm, Nucl. Instrum. Meth. A 611 (2009) 25 [arXiv:0907.3577]. 
[14] T. Behnke et al., The International Linear Collider Technical Design Report - Volume 4: Detectors, arXiv:1306.6329 [physics.ins-det].

[15] H. Abramowicz et al., International Large Detector: Interim Design Report, arXiv:2003.01116 [physics.ins-det].

[16] D. Arominski et al., A detector for CLIC: main parameters and performance, arXiv:1812.07337 [physics.ins-det].

[17] J. Yan et al., Measurement of the Higgs boson mass and $e^{+} e^{-} \rightarrow Z H$ cross section using $Z \rightarrow \mu^{+} \mu^{-}$ and $Z \rightarrow e^{+} e^{-}$at the ILC, Phys. Rev. D 94 (2016) no.11, 113002 [arXiv:1604.07524].

[18] H. Abramowicz et al., Higgs physics at the CLIC electron-positron linear collider, Eur. Phys. J. C 77 (2017) 475 [arXiv:1608.07538].

[19] J. de Blas et al., The CLIC Potential for New Physics, CERN Yellow Rep. Monogr. Vol. 3 (2018), arXiv:1812.02093 [hep-ph].

[20] A. Robson and P. Roloff, Updated CLIC luminosity staging baseline and Higgs coupling prospects, arXiv:1812.01644 [hep-ex].

[21] T. Barklow et al., Improved Formalism for Precision Higgs Coupling Fits, Phys. Rev. D 97 (2018) no.5, 053003 arXiv:1708.08912 [hep-ph].

[22] K. Fujii et al., Physics Case for the $250 \mathrm{GeV}$ Stage of the International Linear Collider, arXiv:1710.07621 [hep-ex].

[23] Y. Kato, Probing the dark sector via searches for invisible decays of the Higgs boson at the ILC, POS (EPS-HEP 2019$) 358$ (2020), arXiv:2002.12048.

[24] P. Roloff et al., Double Higgs boson production and Higgs self-coupling extraction at CLIC, arXiv:1901.05897 [hep-ex].

[25] P. Roloff et al., The Compact Linear $e^{+} e^{-}$Collider (CLIC): Physics Potential, arXiv:1812.07986 [hep-ex].

[26] H. Abramowicz et al., Top-Quark Physics at the CLIC Electron-Positron Linear Collider, JHEP 1911 (2019) 003 [arXiv:1807.02441].

[27] K. Nowak and A.F. Żarnecki, Extracting the top-quark mass and Yukawa coupling from the threshold scan at CLIC, Proc. SPIE 11176, Photonics Applications in Astronomy, Communications, Industry, and High-Energy Physics Experiments 2019, 1117648 (2019).

[28] M. Boronat et al., Top quark mass measurement in radiative events at electron-positron colliders, Phys. Lett. B 804 (2020), 135353 [arXiv:1912.01275].

[29] CMS Collaboration, The Phase-2 Upgrade of the CMS Endcap Calorimeter, CERN-LHCC-2017-023.

[30] ATLAS Collaboration, Expected sensitivity of ATLAS to FCNC top quark decays $t \rightarrow Z u$ and $t \rightarrow H q$ at the High Luminosity LHC, ATL-PHYS-PUB-2016-019.

[31] W. Bernreuther et al., CP-violating top quark couplings at future linear $e^{+} e^{-}$colliders, Eur. Phys. J. C 78 (2018) 155 [arXiv:1710.06737].

[32] Y. Wang et al., Search for Light Scalars Produced in Association with a Z boson at the 250 GeV stage of the ILC, POS ( ICHEP 2018 ) 630 (2019).

[33] K. Mekala et al., Sensitivity to invisible Higgs boson decays at CLIC, arXiv:2002.06034 [hep-ex]. 
[34] J. Kalinowski, W. Kotlarski, T. Robens, D. Sokolowska and A. F. Zarnecki, Benchmarking the Inert Doublet Model for $e^{+} e^{-}$colliders, JHEP 1812 (2018) 081 [arXiv:1809.07712].

[35] A. F. Zarnecki, J. Kalinowski, J. Klamka, P. Sopicki, W. Kotlarski, T. Robens and D. Sokolowska, Inert Doublet Model Signatures at Future $e^{+} e^{-}$Colliders, POS (ALPS2019) 010, arXiv:1908.04659 [hep-ph].

[36] J. Kalinowski, W. Kotlarski, T. Robens, D. Sokolowska and A. F. Zarnecki, Exploring Inert Scalars at CLIC, JHEP 1907 (2019) 053 [arXiv:1811.06952].

[37] D. Sokolowska, J. Kalinowski, J. Klamka, P. Sopicki, A. F. Zarnecki, W. Kotlarski and T. Robens, Inert Doublet Model signatures at future $e^{+} e^{-}$colliders, POS (EPS-HEP 2019$) 570$, arXiv:1911.06254.

[38] A. F. Zarnecki, J. Kalinowski, J. Klamka, P. Sopicki, W. Kotlarski, T. Robens and D. Sokolowska, Searching Inert Scalars at Future $e^{+} e^{-}$Colliders, arXiv:2002.11716 [hep-ph], also submitted to POS (CORFU2019) 047 (these proceedings).

[39] S. Y. Choi, T. Han, J. Kalinowski, K. Rolbiecki and X. Wang, Characterizing invisible electroweak particles through single-photon processes at high energy $e^{+} e^{-}$colliders, Phys. Rev. D 92, no. 9, 095006 (2015) [arXiv:1503.08538].

[40] J. Kalinowski, Single-photon processes at $e^{+} e^{-}$colliders, talk given at CLIC Workshop 2016, https://indico.cern.ch/event/449801/contributions/1945420/

[41] M. Habermehl, Dark Matter at the International Linear Collider, DESY-THESIS-2018-039 (2018).

[42] M. Habermehl, M. Berggren and J. List, WIMP Dark Matter at the International Linear Collider, accepted for publication in Phys. Rev. D, arXiv:2001.03011 [hep-ex]. 